Rev. Int. Contam. Ambie. 37, 319-327, 2021

https://doi.org/10.20937/RICA.53921

\title{
EVALUACIÓN ECONÓMICA DE EVAPORACIÓN DE SALMUERAS DE ÓSMOSIS INVERSA: GESTIÓN AMBIENTAL EN MÉXICO
}

Economic evaluation of reverse osmosis brine evaporation: environmental management in Mexico

\author{
María Isela ENCINAS GUZMÁN, Adriana ROBLES LIZÁRRAGA, \\ Jorge RODRÍGUEZ LÓPEZ y Germán Eduardo DÉVORA ISIORDIA*
}

Departamento de Ciencias del Agua y Medio Ambiente, Instituto Tecnológico de Sonora, Calle 5 de Febrero 818 Sur, Col. Centro, 85000 Ciudad Obregón, Sonora, México

*Autor para correspondencia: german.devora@itson.edu.mx

(Recibido: marzo de 2020; aceptado: julio de 2020)

Palabras clave: escasez de agua, desalinización, vertidos salinos, reducción de residuos, sustentabilidad

\section{RESUMEN}

La desalinización por ósmosis inversa se encuentra dentro de las tecnologías más utilizadas para disminuir la escasez mundial de agua. Sin embargo, presenta impactos ambientales por efluentes de salmuera que no reciben tratamiento previo a su eliminación. El objetivo de este trabajo es evaluar económicamente la evaporación de vertidos de salmueras de procesos de desalinización en agua salobre $(26816 \mathrm{mg} / \mathrm{L}$ sólidos disueltos totales [SDT]) y marina ( $55648 \mathrm{mg} / \mathrm{L} \mathrm{SDT})$ utilizando energía eléctrica y térmica como fuente de calor. El sistema fue óptimo cuando presentó mayor remoción de sales (97\%) y tasa de evaporación $(3.373 \mathrm{~L} / \mathrm{d})$, así como menor consumo energético $(5.43 \mathrm{kWh})$ y costo de operación ( $\$ 8.54 \mathrm{MXN} / \mathrm{d})$, con un nivel de significancia de $\mathrm{p}=0.05$. El escalamiento del sistema eléctrico a un sistema térmico de destilación instantánea multietapa presenta una inversión de $\$ 703016.00$ MXN con un retorno de inversión de $20 \%$, tiempo de recuperación de 10 años y costo del agua producida de $\$ 37.37$ $\mathrm{MXN} / \mathrm{m}^{3}$. Los sistemas de evaporación de salmuera son socialmente eficientes para la disminución de problemáticas ambientales y económicas provocadas por el manejo inadecuado de efluentes de salmueras.

Key words: water shortage, desalination, saline wastes, waste reduction, sustainability

\begin{abstract}
Reverse osmosis desalination is one of the most widely used technologies to reduce global water shortages. However, it presents environmental impacts from brine effluents that do not receive prior treatment at their disposal. The objective of this study is to evaluate the evaporation of brine discharges from desalination processes in brackish water (26816 mg/L total dissolved solids [TDS]) and seawater (55648 mg / L TDS) using electrical and thermal energy as a source of heat. The system was optimal when it presented higher salt removal $(97 \%)$ and evaporation rate $(3.373 \mathrm{~L} / \mathrm{d})$, as well as lower energy consumption (5.43 kWh) and operating cost $(\$ 8.54 \mathrm{MXN} / \mathrm{d})$, with level of significance $p=0.05$. The extension of the electrical system to a multi-stage flash distillation thermal system presents an investment of $\$ 703016.00 \mathrm{MXN}$ with a return
\end{abstract}


of investment of $20 \%$, recovery time of 10 years, and product water cost of $\$ 37.37$ $\mathrm{MXN} / \mathrm{m}^{3}$. Brine evaporation systems are socially efficient for reducing environmental and economic problems caused by the management of brine effluents.

\section{INTRODUCCIÓN}

El desequilibrio entre la disponibilidad y la demanda de agua se ha convertido en un problema global, impulsado principalmente por el crecimiento poblacional acelerado (Garrone et al. 2019, Li et al. 2020). Regiones como la zona occidental de Estados Unidos, Medio Oriente, el norte de África, el sur de Europa, Australia, el norte de China, entre otros, se encuentran en condiciones de escasez de agua. Además, América Latina y el Caribe cuentan con cuencas contaminadas y suministros inadecuados (García-Sánchez y Güereca 2019, Kahil et al. 2019). De acuerdo con Mikosch et al. (2020), se prevé que la demanda mundial de agua aumente alrededor de $55 \%$ en el periodo 2000-2050, lo cual tendrá como consecuencia que más de 3900 millones de personas vivan en condiciones de estrés hídrico grave.

En México, se estima que alrededor de tres millones de personas no cuentan con acceso al agua potable (Pichel et al. 2020). Anualmente el país cuenta con alrededor 460 mil millones de $\mathrm{m}^{3}$ de agua dulce $\mathrm{y}$, de acuerdo con la Organización de las Naciones Unidas (ONU), es considerado con recurso hídrico insuficiente (Muñoz y Becerril 2014). El estado de Sonora, ubicado en el noroeste del territorio mexicano, presenta temperaturas altas y escasa precipitación en aproximadamente $95 \%$ de su superficie; además, la distribución del agua no es equitativa, puesto que el sector agrícola consume $93 \%$ del recurso hídrico total disponible (Ojeda et al. 2014).

Ante esta problemática, la desalinización se ha convertido en una alternativa para disminuir la escasez hídrica, ya que permite tratar agua de alta concentración salina como el agua de mar, la cual representa alrededor del $97 \%$ del recurso hídrico disponible en el mundo (Anis et al. 2019, Manju y Sagar 2017). La desalinización por ósmosis inversa es la tecnología más utilizada globalmente, debido a que en el mundo se obtienen en promedio alrededor de $53 \%$ de agua potable anualmente/diariamente a partir de este proceso de membranas (Saleem y Zaidi 2020).

Sin embargo, aunque la desalinización ofrece muchos beneficios sociales, económicos y de salud pública, esta técnica presenta impactos ambientales significativos (Mannan et al. 2019). El vertido de salmuera en las plantas desalinizadoras es un pro- blema crítico que puede generar la degradación de hábitats marinos, eutrofización costera y cambios puntuales en la calidad del agua de mar, entre otros (Heck et al. 2018).

Por lo tanto, se han desarrollado técnicas de gestión de salmuera, tales como la descarga en mar y tierra, electrodiálisis, inyección de pozos, evaporación, entre otros (Mavukkandy et al. 2019). Algunos de los métodos más utilizados para la evaporación de salmuera son disposición en tanques de evaporación y uso de tecnologías como concentrador de salmuera (BC), destilación instantánea de etapas múltiples (MSF), destilación de efectos múltiples (MED) y evaporación intensificada asistida por el viento (WAIV) (Panagopoulos et al. 2019).

El objetivo de esta investigación es evaluar la demanda económica en procesos de evaporación de vertidos de salmuera proveniente de ósmosis inversa, como método para la gestión ambiental. No obstante que la desalinización por ósmosis inversa es una tecnología acreditada para elevar la oferta de agua dulce, de no recibir tratamiento previo las descargas de salmuera al entorno inmediato, genera impactos ambientales, sociales y económicos.

\section{MATERIALES Y MÉTODOS}

\section{Zona de estudio}

Las pruebas se realizaron en las instalaciones del Laboratorio de Aguas Marinas y Salobres con empleo de Energías Renovables, ubicado en el Instituto Tecnológico de Sonora, en Ciudad Obregón, Sonora (27 29'36.5” N y $109^{\circ} 58^{\prime}$ '11.8” O) (Fig. 1).

\section{Preparación de la muestra}

Se prepararon dos muestras de agua con concentración de 26816 y $55648 \mathrm{mg} / \mathrm{L}$ de sólidos disueltos totales (SDT), las cuales corresponden a las corrientes típicas de rechazo de una planta desalinizadora por ósmosis inversa de $10 \mathrm{~L} / \mathrm{min}$, alimentada con agua salobre y marina (Cuadro I).

\section{Pruebas de evaporación de salmuera}

Se operó un equipo de destilación simple, al cual se agregó un sistema de enfriamiento para mantener el agua del condensador a una temperatura de $2{ }^{\circ} \mathrm{C}$, 


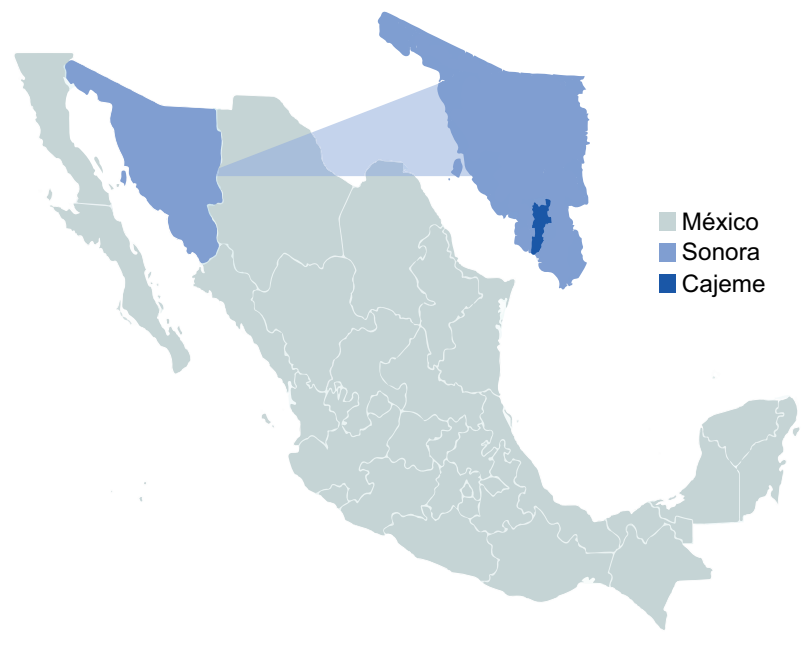

Fig. 1. Ubicación del Laboratorio de Aguas Marinas y Salobres con empleo de Energías Renovables.

aproximadamente. La evaporación se evaluó por medio de dos fuentes de calor, sistema de energía térmica y sistema de energía eléctrica (Fig. 2).

En ambos sistemas se realizaron pruebas utilizando agua a 26816 y $55648 \mathrm{mg} / \mathrm{L}$ de SDT. Con cada una de las concentraciones se llevaron a cabo 10 repeticiones con muestras de $100 \mathrm{~mL}$. En todas las pruebas se midió el tiempo (s) y el volumen del condensado $(\mathrm{mL})$; además, se evaluó la conductividad eléctrica $(\mathrm{mS} / \mathrm{cm})$ por medio de un equipo multiparamétrico YSI 556.

Durante las pruebas de energía eléctrica se midió el consumo energético de la manta térmica y la bomba del sistema de enfriamiento, empleando vatímetros TS-386 de la marca Floureon. Por otra parte, la medición del consumo de gas LP en la evaporación de salmuera mediante energía térmica se obtuvo con la siguiente ecuación:

$V=Q * T$

donde $V$ representa el volumen de gas LP utilizado en cada prueba $\left(\mathrm{m}^{3}\right), Q$ el caudal del flujo de gas $\mathrm{LP}\left(\mathrm{m}^{3} / \mathrm{s}\right)$, el cual siempre se mantuvo constante, $\mathrm{y}$ $T$ (s) el tiempo en que se evaporaron los $100 \mathrm{~mL}$ de mezcla.

Para obtener el caudal se midió la velocidad del gas con un termo-anemómetro de la marca Extech Instruments modelo SDL350; además, se determinó

CUADRO I. CONCENTRACIÓN DE LAS CORRIENTES DEL PROCESO DE DESALINIZACIÓN.

\begin{tabular}{|c|c|c|c|}
\hline \multirow{2}{*}{ Origen del agua } & \multicolumn{3}{|c|}{ Concentración de SDT (mg/L) } \\
\hline & Alimentación & Permeado & Rechazo \\
\hline Salobre & $15739.36 \pm 106.02$ & $1792.47 \pm 36.62$ & $26288.08 \pm 175.31$ \\
\hline Mar & $36033.94 \pm \quad 3.71$ & $4631.21 \pm 449.80$ & $54274.17 \pm 624.72$ \\
\hline
\end{tabular}

SDT: sólidos disueltos totales.

Fuente: Larraguíbel 2018.

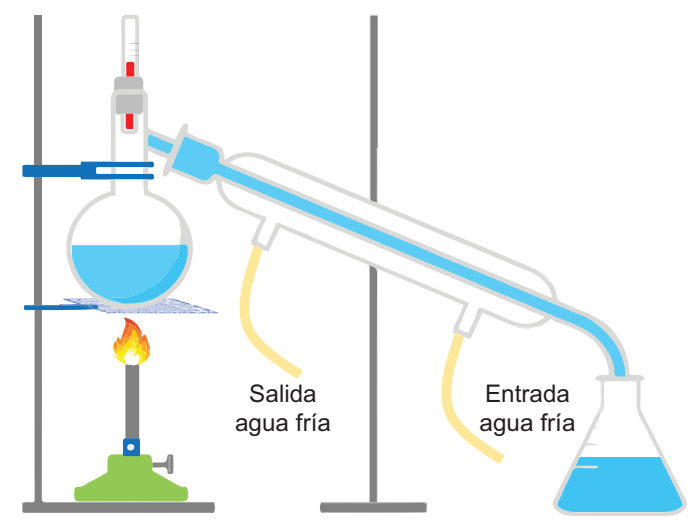

a) Sistema térmico

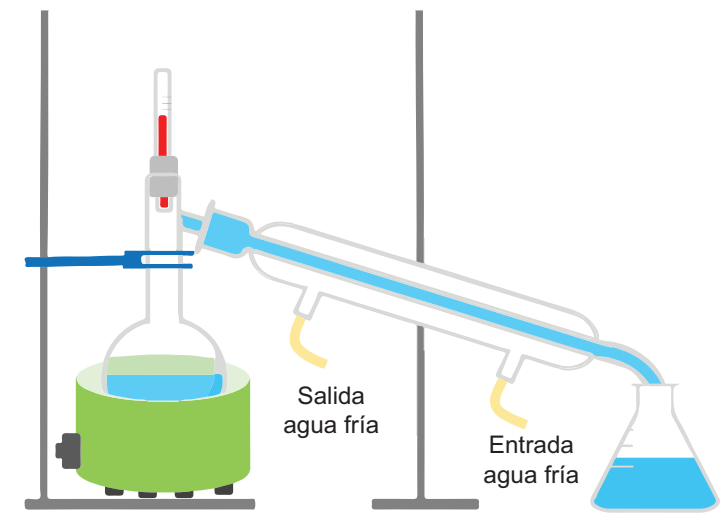

b) Sistema eléctrico

Fig. 2. Sistemas de destilación simple. 
el diámetro del mechero utilizando un calibrador vernier electrónico de la marca Truper; por último, con la finalidad de comparar los sistemas, se obtuvo el consumo energético del sistema térmico considerando $26.9984 \mathrm{kWh} / \mathrm{m}^{3}$ como el poder calorífico del gas LP, establecido por la Norma Oficial Mexicana NOM-027-SCFI-1993 (SECOFI 1993).

\section{Análisis estadístico}

Se utilizó el software Minitab 19 para evaluar la confiabilidad y diferencia significativa de los datos obtenidos en las pruebas, mediante gráficas de probabilidad individuales, gráficas de caja y pruebas $t$ de hipótesis, todas con un valor $\mathrm{p}=.05$.

\section{Selección del proceso más rentable}

Para seleccionar el sistema más eficiente se consideró un día de operación de forma continua; posteriormente se seleccionó el sistema de evaporación de salmuera que presentó mayor remoción de sales (\%) y tasa de evaporación (L/d), así como menor consumo energético $(\mathrm{kWh})$ y costo de operación $(\$ / \mathrm{d})$.

\section{Análisis económico}

Se utilizó el método planteado por Pérez (2017) para estimar el valor de la inversión a escala industrial, tomando en cuenta el proceso de MSF, debido a que es la tecnología más utilizada dentro de los procesos térmicos de desalinización y la que presentó mayor similitud respecto al desarrollo experimental (Choi 2016). Sin embargo, se consideró una sola etapa, la cual consiste principalmente en evaporación y destilación. Posteriormente se seleccionaron aquellos equipos que presentaron mayor similitud en cuanto a diseño y función, respecto a los utilizados en el laboratorio, siendo éstos un evaporador (A), condensador (B), tanque de alimentación (C), agua producto (D) y sólidos de salmuera (E) (ecuación 2).

$C T I=F * \sum$ Costo $_{A+B+C+D+E}$

donde $F$ representa el valor del factor de Lang, el cual varía de acuerdo con el producto de la planta, siendo 3.10 para sólidos, 3.63 mezcla de sólidos-fluidos y 4.74 fluidos (Amigun y Blottnitz 2009).

Para la sumatoria de costo de los equipos se seleccionaron un evaporador de tubo largo (ecuación 3 ), un condensador de doble tubo (ecuación 4) y un tanque de techo fijo (ecuación 5) para alimentación, agua producto y sólidos de salmuera, todos de acero inoxidable.

$$
\text { Costo }_{\text {Evaporador }}=\text { Costo }_{\text {equipo }} * F_{M} * F_{P}
$$

$$
\begin{aligned}
& \text { Costo }_{\text {Condensador }}=\text { Costo }_{\text {equipo }} * \\
& \left(B_{1}+B_{2} * F_{M} * F_{P}\right) \\
& \text { Costo }_{\text {Tanque }}=\text { Costo }_{\text {equipo }} * \\
& \left(B_{1}+B_{2} * F_{M} * F_{P}\right)
\end{aligned}
$$

En cada una de las ecuaciones planteadas se añadieron factores de corrección para el costo de los equipos $\left(B_{1}\right.$ y $\left.B_{2}\right)$. Fm corresponde al tipo de material (acero inoxidable) y $F p$ al factor por presión; sin embargo, este último no se consideró debido a que todas las pruebas se realizaron en condiciones ambientales. En el cuadro II se muestra un resumen de los factores seleccionados, de acuerdo con datos presentados por Pérez (2017).

CUADRO II. CORRECCIONES PARA COSTO DE EQUIPOS.

\begin{tabular}{lccc}
\hline Equipo & Fm & $\mathrm{B}_{1}$ & $\mathrm{~B}_{2}$ \\
\hline Evaporador tubo largo & 5.08 & - & - \\
Condensador tubo doble & 2.73 & 1.74 & 1.55 \\
Tanques de techo fijo & - & 1.10 & 0.00 \\
\hline
\end{tabular}

Fm: factor del tipo de material, $\mathrm{B}_{1}$ : factor de corrección para costo, $\mathrm{B}_{2}$ : factor de corrección para costo.

Fuente: Pérez 2017.

Una vez estimados los costos económicos, se puede evaluar la rentabilidad económica del sistema seleccionado (Jiménez 2013), la cual incluye elementos como tasa de rendimiento de la inversión (ecuación 6), utilidad neta (ecuación 7), utilidad bruta (ecuación 8), costo de operación anual (ecuación 9) y tiempo de recuperación de capital (ecuación 10). Además, se consideró una planta de ósmosis inversa con capacidad de producción de $37743 \mathrm{~m}^{3} / \mathrm{d}$ y coeficiente de conversión de $45 \%$ (Robles et al. 2020), con la finalidad de obtener una proyección de la cantidad de salmuera que se produciría en un día en escala real.

$$
\begin{aligned}
& R O I=\frac{P}{I} \times 100 \\
& P=R-e I-t(R-d I) \\
& R=S-C
\end{aligned}
$$

$C=a I+b M P+c E+d M O-p S P$ 
$T R=\frac{I}{P+e I}$

\section{RESULTADOS Y DISCUSIÓN}

Los resultados de las pruebas realizadas en Minitab se muestran en el cuadro III, el cual contiene las diferencias significativas obtenidas al comparar la variación de concentración de la salmuera y el tipo de fuente de calor para el sistema de evaporación.

CUADRO III. ANÁLISIS DE DIFERENCIA SIGNIFICATIVA DE LAS PRUEBAS DE EVAPORACIÓN.

\begin{tabular}{lccc}
\hline & $\begin{array}{c}\text { Tasa de } \\
\text { evaporación } \\
(\mathrm{mL} / \mathrm{s})\end{array}$ & $\begin{array}{c}\text { Consumo } \\
\text { energético } \\
(\mathrm{kWh})\end{array}$ & $\begin{array}{c}\text { Eficiencia de } \\
\text { remoción } \\
(\%)\end{array}$ \\
\hline $\begin{array}{l}\text { Concentración } \\
\text { de salmuera }\end{array}$ & $\mathrm{Ha}$ & $\mathrm{Ha}$ & $\mathrm{Ha}$ \\
$\begin{array}{l}\text { Fuente de calor } \\
\mathrm{Ha}\end{array}$ & $\mathrm{Ha}$ & $\mathrm{Ha}$ \\
\hline
\end{tabular}

Todos los parámetros evaluados presentaron diferencias significativas, siendo salinidad y temperatura los factores más importantes. Durante las pruebas se obtuvieron temperaturas de ebullición de 101 y $102{ }^{\circ} \mathrm{C}$, aproximadamente, para salmuera de desalinización de agua salobre y agua de mar, respectivamente. Estos resultados coinciden con el rango de temperaturas en procesos de desalinización con fuentes de calor externas presentado por Zhao et al. (2019), quienes establecen un rango de 90 a $110^{\circ} \mathrm{C}$.

El agua se encuentra dentro de los compuestos con puntos de ebullición más elevados $\left(100^{\circ} \mathrm{C}\right)$, de acuerdo con Chang (2008), lo cual se debe a la extensa formación de puentes de hidrógeno entre moléculas. Además, el aumento en la concentración de sólidos presentes en una mezcla eleva su punto de ebullición (Gordon y Tong 2016). La figura 3 muestra la comparación entre las tasas de evaporación de los sistemas eléctrico y térmico.

El sistema con suministro de energía eléctrica presentó tasas de evaporación de 0.035 y $0.0431 \mathrm{~mL} / \mathrm{s}$ para concentraciones de 26816 y $55648 \mathrm{mg} / \mathrm{L}$ de SDT, respectivamente. Por otra parte, el sistema térmico obtuvo tasas de 0.0346 y $0.0367 \mathrm{mg} / \mathrm{L}$ en salmueras de desalinización salobre y salmueras de desalinización de agua marina, respectivamente. Al variar la concentración del agua la diferencia en la

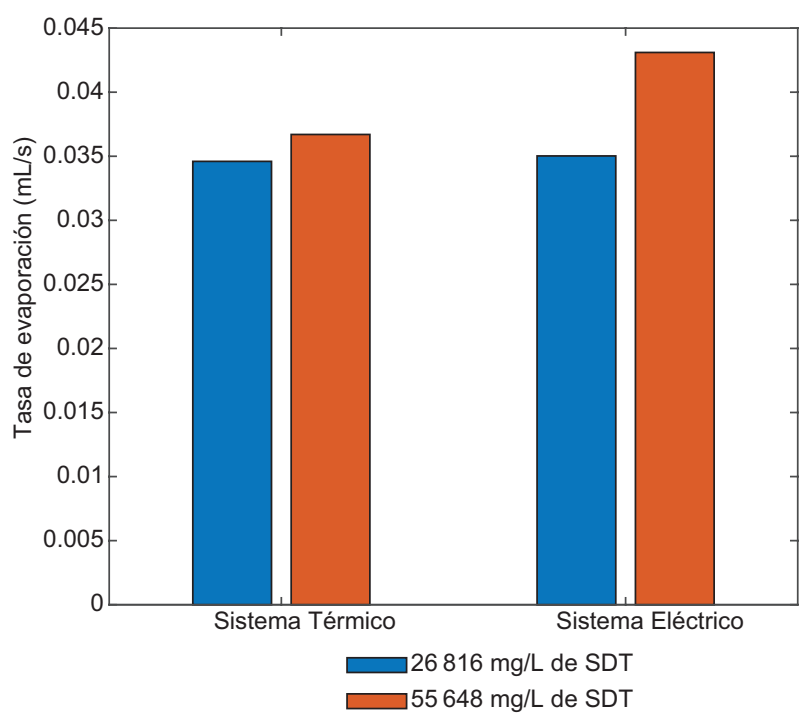

Fig. 3. Comparación de la tasa de evaporación en el sistema térmico y el sistema eléctrico.

tasa de evaporación fue de alrededor de $0.0052 \mathrm{~mL} / \mathrm{s}$, mientras que entre sistemas la diferencia fue menor de $0.003 \mathrm{~mL} / \mathrm{s}$.

Estas diferencias se deben principalmente a la temperatura en la que operaron los sistemas, lo cual coincide con lo reportado por López (2008), quien menciona que la temperatura es el parámetro al que la evaporación se muestra más sensible. Esto se debe en gran parte al rompimiento de los puentes de hidrógeno que ocurre al aumentar la temperatura, lo cual genera un incremento en la energía cinética de las moléculas. Cuando están en estado gaseoso, las moléculas son menos densas y ocupan mayor volumen; por lo tanto, a mayor temperatura mayor será la tasa de evaporación (Chang 2008).

La figura 4 muestra los resultados de la cantidad de energía consumida por cada uno de los sistemas a diferentes concentraciones. $\mathrm{Al}$ aumentar la concentración de SDT en el agua de 26816 a $55648 \mathrm{mg} / \mathrm{L}$, se observó un incremento en el consumo energético de 13.44 y $6.10 \%$ al usar el sistema eléctrico y el sistema térmico, respectivamente. Por lo tanto, se observa una relación entre el aumento de concentración y el incremento de la demanda energética, lo cual coincide con Kim et al. (2019), quienes reportan que la concentración del agua de alimentación es un factor importante debido a que el incremento de salinidad aumenta directamente el consumo energético.

Por otra parte, también se presentaron diferencias al variar la fuente de calor. La demanda energética del sistema térmico fue $1.3 \mathrm{kWh}$ mayor con relación a la 


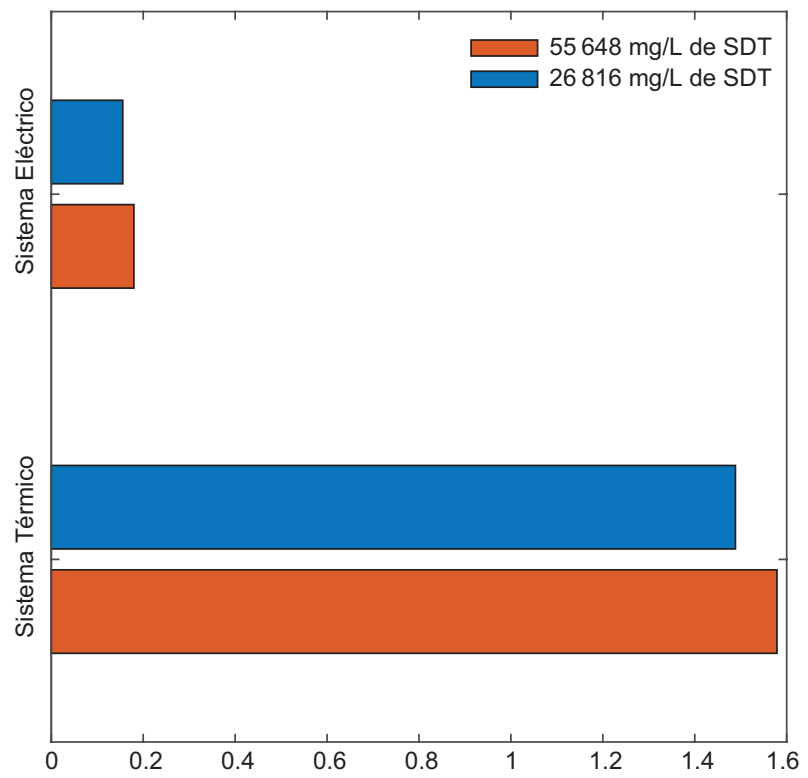

Fig. 4. Consumo energético por sistemas de evaporación.

del sistema eléctrico (75.51\%, aproximadamente). Este resultado es similar a al obtenido por Alhaj y al-Ghamdi (2019), quienes señalan que el consumo de los procesos de desalinización alimentados con energía térmica es alrededor de $95 \%$ mayor que el de los sistemas que utilizan energía eléctrica.

Los resultados de la eficiencia de remoción de SDT se muestran en el cuadro IV. El sistema eléctrico logró remover más del $95 \%$ y la concentración final de SDT fue $25789.73 \mathrm{mg} / \mathrm{L}$ en la salmuera de agua salobre y $54590.36 \mathrm{mg} / \mathrm{L}$ en salmuera de agua de mar. Sin embargo, la remoción en el sistema de energía térmica fue mayor: el agua producto obtenida presentó una concentración de 97.74 y $52.29 \mathrm{mg} / \mathrm{L}$ de SDT, para una alimentación de 26816 y $55648 \mathrm{mg} / \mathrm{L}$ de SDT respectivamente.

Retomando los resultados anteriores, los sistemas lograron reducir la mayor parte de la concentración de SDT que se encontraba presente en las salmueras, presentando entre 96 y $99 \%$ de remoción, lo cual es similar a lo reportado por Panagopoulos et al. (2019), quienes observaron que los tratamientos de evaporación de salmueras que operan con sistemas térmicos presentan un porcentaje de remoción con eficiencia del 85 a $99 \%$.

Existe una diferencia del $3 \%$ en la eficiencia de remoción entre los sistemas evaluados. Sin embargo, en promedio la concentración de SDT en el agua producto empleando el sistema eléctrico fue de 1041.95 y de $75.02 \mathrm{mg} / \mathrm{L}$ para el sistema térmico, lo cual de acuerdo con la clasificación de agua reportada por Dévora-Isiordia et al. (2013), las clasifica como aguas salobre y potable, respectivamente.

\section{Selección del sistema}

Se seleccionó el sistema eléctrico como el sistema de evaporación de salmuera más eficiente, ya que presenta $75 \%$ de las condiciones óptimas establecidas (Cuadro V). A pesar de que el sistema con empleo de energía térmica presentó mayor porcentaje de remoción, el empleo de energía eléctrica resultó ser más eficiente en cuanto a consumo energético $(\mathrm{kWh})$, tasa de evaporación (L/d) y costo de operación $(\$ / \mathrm{kWh})$; además, la eficiencia de remoción se encuentra dentro de los valores reportados para sistemas térmicos.

\section{Análisis económico}

Con el método basado en el estudio de Pérez (2017) se obtuvo una inversión de $\$ 703016.00$ MXN, la cual está compuesta por la sumatoria del costo total del evaporador (\$92991.22 MXN), condensador ( $\$ 93126.18 \mathrm{MXN}$ ) y los tanques de alimentación, agua producto y sólidos $(\$ 2,516.99$ MXN c/u). Posteriormente, de acuerdo con la planta de ósmosis inversa reportada por Robles et al. (2020), se obtuvo un valor de $46130 \mathrm{~m}^{3} / \mathrm{d}$ en la corriente de rechazo, una tasa de retorno (ROI) de $20 \%$ y un tiempo de recuperación de 10 años, considerando la inversión y los valores de los componentes

CUADRO IV. COMPARACIÓN DE LA EFICIENCIADE REMOCIÓN DE SDT POR SISTEMA Y TIPO DE AGUA.

\begin{tabular}{lcccccc}
\hline \multicolumn{3}{c}{ Sistema de energía eléctrica } & & \multicolumn{3}{c}{ Sistema de energía térmica } \\
\cline { 1 - 2 } \cline { 5 - 7 } $\begin{array}{l}\text { Alimentación } \\
(\mathrm{mg} / \mathrm{L})\end{array}$ & $\begin{array}{c}\text { Producto } \\
(\mathrm{mg} / \mathrm{L})\end{array}$ & $\begin{array}{c}\text { Eficiencia de } \\
\text { remoción }(\%)\end{array}$ & & $\begin{array}{c}\text { Alimentación } \\
(\mathrm{mg} / \mathrm{L})\end{array}$ & $\begin{array}{c}\text { Producto } \\
(\mathrm{mg} / \mathrm{L})\end{array}$ & $\begin{array}{c}\text { Eficiencia de } \\
\text { remoción }(\%)\end{array}$ \\
\hline 26816.00 & 1026.27 & 96.17 & & 26816.00 & 52.29 & 99.90 \\
55648.00 & 1057.64 & 98.10 & & 55648.00 & 97.74 & 99.65 \\
\hline
\end{tabular}


CUADRO V. COMPARACIÓN FINAL DE LOS RESULTADOS OBTENIDOS.

\begin{tabular}{lccccc}
\hline \multirow{2}{*}{ Parámetro } & \multicolumn{2}{c}{ Sistema de energía eléctrica } & & \multicolumn{2}{c}{ Sistema de energía térmica } \\
\cline { 2 - 3 } \cline { 5 - 6 } & $26816 \mathrm{mg} / \mathrm{L}$ & $55648 \mathrm{mg} / \mathrm{L}$ & & $26816 \mathrm{mg} / \mathrm{L}$ & $55648 \mathrm{mg} / \mathrm{L}$ \\
\hline Consumo energético $(\mathrm{kWh})$ & 5.43 & 5.78 & & 46.88 & 49.73 \\
Tasa de evaporación $(\mathrm{L} / \mathrm{d})$ & 3.03 & 3.72 & & 2.99 & 3.17 \\
Eficiencia de remoción $(\%)$ & 96.17 & 98.10 & & 99.65 & 99.60 \\
Costo de operación $(\$ / \mathrm{d})$ & 8.28 & 8.80 & & 31.37 & 33.26 \\
\hline
\end{tabular}

económicos del proceso mostrados en el cuadro VI. De acuerdo con Jiménez (2003), un proceso se considera rentable cuando presenta una $\mathrm{ROI} \geq 20 \%$.

\section{CUADRO VI. COMPONENTES DE LA ECONOMÍA DEL PROCESO.}

\begin{tabular}{lr}
\hline Componentes & \multicolumn{1}{c}{ \$/año } \\
\hline Costo de operación (C) & $629248689.46 \mathrm{MXN}$ \\
Ventas anuales (S) & $29600197.56 \mathrm{MXN}$ \\
Utilidad bruta (R) & $351508.09 \mathrm{MXN}$ \\
Utilidad neta (P) & $140603.24 \mathrm{MXN}$ \\
\hline
\end{tabular}

Por otra parte, el costo del agua obtenido con la proyección para evaporar la salmuera proveniente del proceso de desalinización de ósmosis inversa fue de $\$ 37.37 \mathrm{MXN} / \mathrm{m}^{3}$ (\$1.93 $\left.\mathrm{USD} / \mathrm{m}^{3}\right)$, similar al obtenido por al-Hamahmy et al. (2016), quienes reportan un costo del agua en una planta de MSF convencional de aproximadamente $\$ 23.49 \mathrm{MXN} / \mathrm{m}^{3}$ $\left(\$ 1.205 \mathrm{USD} / \mathrm{m}^{3}\right)$.

\section{CONCLUSIONES}

Se evaluó la eficiencia energética y económica de un proceso de evaporación de salmuera en condiciones controladas y a escala laboratorio, mediante la variación de la concentración (25 789 y $54590.36 \mathrm{mg} / \mathrm{L}$ SDT) y fuente de calor suministrada (energía térmica y eléctrica). El sistema eléctrico se consideró más eficiente, ya que logró remover $97 \%$ de los sólidos disueltos totales, con una tasa de evaporación de $3.373 \mathrm{~L} / \mathrm{d}$, demanda energética de $5.43 \mathrm{kWh}$ y un costo de producción de $\$ 8.54 \mathrm{MXN} / \mathrm{d}$, con un valor de significancia de $\mathrm{p}=0.05$. El escalamiento del sistema eléctrico a un sistema térmico MSF presenta una inversión de $\$ 703016.00 \mathrm{MXN}$ considerando los equipos más importantes.
El análisis económico demostró que el proceso es rentable, con un ROI de $20 \%$ y tiempo de recuperación de 10 años, costo de operación anual de \$629248689.46 MXN/año, ventas anuales de \$629600 197.56 MXN/año, utilidad bruta de \$351 508.09 MXN/año y utilidad neta de \$140603.24 MXN/año, siendo de $\$ 37.37 \mathrm{MXN} / \mathrm{m}^{3}$ el costo del agua producto obtenida del proceso de evaporación.

La eliminación de salmueras que no reciben tratamiento previo pueden impactar negativamente el medio ambiente, principalmente a los ecosistemas marinos. Por lo tanto, es importante generar investigaciones que brinden alternativas para el manejo de la salmuera como un recurso y no sólo como residuo. En este sentido, los sistemas de evaporación de salmuera son técnicas ecoeficientes después de aplicar la tecnología de ósmosis inversa en los procesos de desalinización, ya que permiten abatir problemas ambientales y económicos provocados por el manejo incorrecto de estos efluentes.

\section{AGRADECIMIENTOS}

Los autores agradecen al Instituto Tecnológico de Sonora por el financiamiento del proyecto a través el programa de apoyo a la investigación PROFAPI.

\section{REFERENCIAS}

Al-Hamahmy M., Fath H. y Khanafer K. (2016). Technoeconomical simulation and study of a novel MSF desalination process. Desalination 386, 1-12. https:// doi.org/10.1016/j.desal.2016.02.018

Alhaj M. y al-Ghamdi S. (2019). Why is powering thermal desalination with concentrated solar power expensive? Assessing economic feasibility and market commercialization barriers. Sol. Energy. 189, 480-490. https:// doi.org/10.1016/j.solener.2019.07.046

Amigun B. y Blottnitz H. (2009). Cost analyses and predictions for a fuel ethanol plant in a rural and landlocked 
African country: Lang factor approach. Int. J. Prod. Econ. 119 (1), 207-216. https://doi.org/10.1016/j. ijpe.2009.02.006

Anis S., Hashaikeh R. y Hilal N. (2019). Functional materials in desalination: A review. Desalination 468, 114077. https://doi.org/10.1016/j.desal.2019.114077

Chang R. (2008). Fisicoquímica. 3ra ed. McGrawHill, Ciudad de México, México, 1018 pp.

Choi S. (2016). On the brine re-utilization of a multi-stage flashing (MSF) desalination plant. Desalination 398, 64-76. https://doi.org/10.1016/j.desal.2016.07.020

Dévora-Isiordia G., González-Enríquez R. y Ruiz-Cruz S. (2013). Evaluación de procesos de desalinización y su desarrollo en México. Tecnol. Cienc. Agua 4 (3), 27-46.

García-Sánchez M. y Güereca L. (2019). Environmental and social life cycle assessment of urban water systems: The case of Mexico City. Sci. Total. Environ. 693. https://doi.org/10.1016/j.scitotenv.2019.07.270

Garrone P., Grilli L. y Marzano R. (2019). Price elasticity of water demand considering scarcity and attitudes. Util. Policy. 59. https://doi.org/10.1016/j. jup.2019.100927

Gordon J. y Tong H. (2016). Thermodynamics perspective for the specific energy consumption of seawater desalination. Desalination 386, 13-18. https://doi. org/10.1016/j.desal.2016.02.030

Heck N., Paytan A., Potts D., Haddad B. y Petersen L. (2018). Management preferences and attitudes regarding environmental impacts from seawater desalination: Insights from a small coastal community. Ocean. Coastal. Manage. 163, 22-29. https://doi. org/10.101Llópez6/j.ocecoaman.2018.05.024

Jiménez, A. (2013). Diseño de procesos en ingeniería química. Editorial Reverté, Celaya, México, 256 pp.

Kahil T., Albiac J., Fischer G., Strokal M., Tramberend S., Greve P., Tang T., Burek P., Burtscher R. y Wada Y. (2019). A nexus modeling framework for assessing water scarcity solutions. Curr. Opin. Env. Sust. 40, 72-80. https://doi.org/10.1016/j.cosust.2019.09.009

Kim J., Park K., Yang D. y Hong, S. (2019). A comprehensive review of energy consumption of seawater reverse osmosis desalination plants. Appl. Energ. 254. https:// doi.org/10.1016/j.apenergy.2019.113652

Larraguíbel O. (2018). Evaluación del consumo energético de una planta desalinizadora por osmosis inversa acoplada a un sistema fotovoltaico con seguidor de dos ejes. Tesis de Licenciatura. Instituto Tecnológico de Sonora. Ciudad Obregón, México. 80 pp.

Li W., Hai X., Han L., Mao. y Tian M. (2020). Does urbanization intensify regional water scarcity? Evidence and implications from a megaregion of China. J. Clean. Prod. 244. https://doi.org/10.1016/j. jclepro.2019.118592
López J. (2008). Estimación de las tasas de evaporación de los depósitos pirenaicos. Cuadernos de Investigación Geográfica 34, 61-81. https://doi.org/10.18172/cig.1207

Manju S. y Sagar N. (2017). Renewable energy integrated desalination: A sustainable solution to overcome future fresh-water scarcity in India. Renew. Sust. Energ. Rev. 73, 594-609. https://doi.org/10.1016/j. rser.2017.01.164

Mannan M., Alhaj M., Mabrouk A. y al-Ghamdi S. (2019). Examining the life-cycle environmental impacts of desalination: A case study in the State of Qatar. Desalination 452, 238-246. https://doi.org/10.1016/j. desal.2018.11.017

Mavukkandy M., Chabib C., Mustafa I., Ghaferi A. y AlMarzooqui F. (2019). Brine management in desalination industry: From waste to resources generation. Desalination 472. https://doi.org/10.1016/j.desal.2019.114187

Mikosch N., Becker R., Schelter L., Berger M., Usman M. y Finkbeiner M. (2020). High resolution water scarcity analysis for cotton cultivation areas in Punjab, Pakistan. Ecol. Indic. Volume 109, 105852. https://doi. org/10.1016/j.ecolind.2019.105852

Muñoz F. y Becerril L. (2014). Low-capacity reverse osmosis solar desalination plant. Energ. Proced. 57, 27872793. https://doi.org/10.1016/j.egypro.2014.10.311

Ojeda A., Narváez A. y Pacheco J. (2014). Gestión del agua doméstica urbana en Hermosillo (Sonora, México). Cuad. Geogr. Rev. Colomb. Geogr. 23 (1), 147. http:// dx.doi.org/10.15446/rcdg.v23n1.41089

Panagopoulos A., Haralambous K. y Loizidou M. (2019). Desalination brine disposal methods and treatment technologies - A review. Sci. Total. Environ. 693. https://doi.org/10.1016/j.scitotenv.2019.07.351

Pérez M. (2017). Diseño de una planta desalinizadora de 20,000 $\mathrm{m}^{3}$ /día basada en una tecnología de desalinización multiefecto (MED). Tesis de licenciatura. Escuela Técnica Superior de Ingeniería, Universidad de Sevilla, Sevilla, España, 135 pp.

Pichel N., Vivar M., Fuentes M. y Eugenio-Cruz K. (2020). Study of a hybrid photovoltaic-photochemical technology for meeting the needs of safe drinking water and electricity in developing countries: First field trial in rural Mexico. Journal of Water Process Engineering 33, 101056. https://doi.org/10.1016/j.jwpe.2019.101056

Robles A., Martínez M., Encinas M., Larraguíbel O., Rodríguez J. y Dévora G. (2020). Design of reverse osmosis desalination plant in Puerto Peñasco, Sonora, México. Desalin. Water. Treat. 175, 1-10. https://doi. org/10.5004/dwt.2020.24739

Saleem H. y Zaidi S. (2020). Nanoparticles in reverse osmosis membranes for desalination: A state of the art review. Desalination 475. https://doi.org/10.1016/j. desal.2019.114171 
SECOFI (1993). Norma Oficial Mexicana NOM-027-SCFI-1993. Calentadores para agua tipo almacenamiento a base de gases licuados de petróleo o gas natural. Secretaría de Comercio y Fomento Industrial. Diario Oficial de la Federación, 9 de julio.
Zhao D., Lee L., Ong L., Chowdhry P., Siah K. y Ng Y. (2019). Electrodialysis reversal for industrial reverse osmosis brine treatment. Sep. Purif Technol. 213, 339-347. https://doi.org/10.1016/j.seppur.2018.12.056 